\title{
Monomeric eNAMPT in the development of experimental diabetes in mice: a potential target for type 2 diabetes treatment
}

\author{
Julius Kieswich $^{1}$ - Sophie R. Sayers ${ }^{2}$ - Marta F. Silvestre ${ }^{3,4}$ - Steven M. Harwood ${ }^{1}$. \\ Muhammad M. Yaqoob ${ }^{1} \cdot$ Paul W. Caton ${ }^{2}$
}

Received: 6 May 2016 / Accepted: 22 July 2016/Published online: 19 August 2016

(C) The Author(s) 2016. This article is published with open access at Springerlink.com

\begin{abstract}
Aims/hypothesis Serum extracellular nicotinamide phosphoribosyltransferase (eNAMPT) concentrations are elevated in type 2 diabetes. However, the relationship between abnormally elevated serum eNAMPT and type 2 diabetes pathophysiology is unclear. eNAMPT circulates in functionally and structurally distinct monomeric and dimeric forms. Dimeric eNAMPT promotes NAD biosynthesis. The role of eNAMPT-monomer is unclear but it may have NADindependent proinflammatory effects. However, studies of eNAMPT in type 2 diabetes have not distinguished between monomeric and dimeric forms. Since type 2 diabetes is characterised by chronic inflammation, we hypothesised a selective NAD-independent role for eNAMPT-monomer in type 2 diabetes.

Methods Two mouse models were used to examine the role of eNAMPT-monomer in type 2 diabetes; (1) a mouse model of diabetes fed a high-fat diet (HFD) for 10 weeks received i.p.
\end{abstract}

Electronic supplementary material The online version of this article (doi:10.1007/s00125-016-4076-3) contains peer-reviewed but unedited supplementary material, which is available to authorised users.

Paul W. Caton

paul.w.caton@kcl.ac.uk

1 Translational Medicine and Therapeutics, William Harvey Research Institute, Bart's and the London School of Medicine and Dentistry, Queen Mary University of London, London, UK

2 Diabetes Research Group, Division of Diabetes and Nutritional Sciences, King's College London, Hodgkin Building, Guy's Campus, London SE1 1UL, UK

3 Faculdade de Medicina da Universidade de Lisboa, Lisbon, Portugal

4 Human Nutrition Unit, University of Auckland, Auckland, New Zealand injections with an anti-monomeric-eNAMPT antibody; and (2) lean non-diabetic mice received i.p. injections with recombinant monomeric eNAMPT daily for 14 days.

Results Serum monomeric eNAMPT levels were elevated in HFD-fed mouse models of diabetes, whilst eNAMPT-dimer levels were unchanged. eNAMPT-monomer neutralisation in HFD-fed mice resulted in lower blood glucose levels, amelioration of impaired glucose tolerance (IGT) and whole-body insulin resistance, improved pancreatic islet function, and reduced inflammation. These effects were maintained for at least 3 weeks post-treatment. eNAMPT-monomer administration induced a diabetic phenotype in mice, characterised by elevated blood glucose, IGT, impaired pancreatic insulin secretion and the presence of systemic and tissue inflammation, without changes in NAD levels.

Conclusions/interpretation We demonstrate that elevation of monomeric-eNAMPT plays an important role in the pathogenesis of diet-induced diabetes via proinflammatory mechanisms. These data provide proof-of-concept evidence that the eNAMPT-monomer represents a potential therapeutic target for type 2 diabetes.

Keywords Beta cell $\cdot$ Extracellular nicotinamide phosphoribosyltransferase $\cdot$ eNAMPT $\cdot$ Inflammation · Islet · Type 2 diabetes

$\begin{array}{ll}\text { Abbreviations } \\ \text { AT } & \text { Adipose tissue } \\ \text { CCL2 } & \text { Chemokine (C-C motif) ligand 2 } \\ \text { CON } & \text { Control diet } \\ \text { CON }^{\mathrm{Ab}} & \begin{array}{l}\text { Control diet-fed mice, administered eNAMPT- } \\ \text { antibody }\end{array} \\ \mathrm{CON}^{\mathrm{IgG}} & \begin{array}{l}\text { Control diet-fed mice, administered IgG } \\ \text { HFD }\end{array} \\ \text { High-fat diet }\end{array}$




\begin{tabular}{|c|c|}
\hline $\mathrm{HFD}^{\mathrm{Ab}}$ & $\begin{array}{l}\text { High-fat diet-fed mice, administered eNAMPT } \\
\text { antibody }\end{array}$ \\
\hline $\mathrm{HFD}^{\mathrm{IgG}}$ & High-fat diet-fed mice, administered IgG \\
\hline IGT & Impaired glucose tolerance \\
\hline iNAMPT & $\begin{array}{l}\text { Intracellular nicotinamide } \\
\text { phosphoribosyltransferase }\end{array}$ \\
\hline eNAMPT & $\begin{array}{l}\text { Extracellular nicotinamide } \\
\text { phosphoribosyltransferase }\end{array}$ \\
\hline GSIS & Glucose-stimulated insulin secretion \\
\hline HFD & High-fat diet \\
\hline iNAMPT & $\begin{array}{l}\text { Intracellular nicotinamide } \\
\text { phosphoribosyltransferase }\end{array}$ \\
\hline MCP1 & Monocyte chemoattractant protein \\
\hline NAFLD & Non-alcoholic fatty liver disease \\
\hline NAMPT & Nicotinamide phosphoribosyltransferase \\
\hline NMN & Nicotinamide mononucleotide \\
\hline PBEF & Pre-B cell colony-enhancing factor \\
\hline qRT-PCR & Quantitative RT-PCR \\
\hline SVF & Stromal vascular fraction \\
\hline
\end{tabular}

\section{Introduction}

Type 2 diabetes is characterised by the presence of peripheral insulin resistance and pancreatic beta cell dysfunction [1]. Determining the precise pathophysiological mechanisms responsible for these processes is essential for the development of novel therapeutics.

Serum concentrations of extracellular nicotinamide phosphoribosyltransferase (eNAMPT; also referred to as visfatin/pre-B cell colony-enhancing factor [PBEF]) are commonly elevated in type 2 diabetes patients [2], whilst raised eNAMPT levels strongly correlate with declining beta cell function [3]. Therefore, a pathophysiological role is implied for eNAMPT in type 2 diabetes. However, other studies have reported both insulin sensitising and beta cell protective effects of eNAMPT [4-7]. Therefore, the precise relationship between elevated eNAMPT and type 2 diabetes remains unresolved.

Nicotinamide phosphoribosyltransferase exists in intracellular (iNAMPT) and extracellular (eNAMPT) forms. iNAMPT is widely expressed and is a well-characterised NAD biosynthetic enzyme [8]. In contrast, the function of eNAMPT is unclear, although putative proinflammatory [9, 10], insulin-mimetic [11] and NAD biosynthetic functions $[5,12,13]$ have been described. These disparate putative functions are controversial and have been challenged [11, 14], but may be explained by the presence of structurally and functionally distinct monomeric $(50 \mathrm{kDa})$ and dimeric $(100 \mathrm{kDa})$ forms of eNAMPT. Dimerisation is reportedly essential for the biosynthetic functions of NAMPT [5, 15]. The eNAMPT-monomer has potential NAD-independent proinflammatory effects. However, the precise structure-function relationships have not yet been fully investigated, particularly within the context of type 2 diabetes pathophysiology.

Given the crucial role of chronic inflammation in type 2 diabetes pathophysiology, we hypothesised that eNAMPTmonomer levels will be selectively elevated in type 2 diabetes and by potentially acting in a proinflammatory manner, may play a key role in type 2 diabetes pathophysiology.

\section{Methods}

Animal studies For immunoneutralisation experiments, 8-week-old male C57Bl/6 mice (Charles River, Margate, UK) were fed a high-fat diet (HFD; 60\% wt/wt fat; 58Y1; Test Diets, St. Louis, MO, USA) or a control diet (CON) for 10 or 13 weeks, and then injected i.p. with a rabbit polyclonal mouse anti-eNAMPT antibody $(2.5 \mu \mathrm{g} / \mathrm{ml}$; LS-C48964; LifespanBio, WA, USA) or a non-immune IgG equivalent (four separate doses during weeks 9-10). Antibodies were validated via immunoprecipitation and immunoblotting. Mice were then killed either directly post-treatment (at 10 weeks) or 3 weeks later (at 13 weeks; $n=24$ /group)

For experimental elevation of eNAMPT and nicotinamide mononucleotide (NMN), 8-week old male C57Bl/6 mice were i.p. injected daily with either recombinant eNAMPT ( $5 \mathrm{ng} / \mathrm{ml}$; Adipogen, Seoul, South Korea), NMN (500 mg/kg body weight; Sigma, Poole, UK) or the equivalent volume of $\mathrm{NaCl} 154 \mathrm{mmol} / \mathrm{l}$ (Sigma) for 14 days ( $n=8 /$ group).

Mice were maintained on a $12 \mathrm{~h}$ light/ $12 \mathrm{~h}$ dark cycle. Animal experiments were conducted in accordance with UK Home Office Animals (Scientific Procedures) Act 1986, with local ethical committee approval. Experimenters were not blinded to group assignment or outcome assessment. Specific randomisation of animals into groups was not carried out. No data, samples or animals were excluded from the study.

IPGTT and in vivo insulin secretion Mice were fasted overnight then injected i.p. with $2 \mathrm{~g} / \mathrm{kg}$ body weight $25 \% \mathrm{wt} / \mathrm{vol}$. dextrose (Sigma). Glucose was measured in tail vein blood samples (Accuchek, Roche Diagnostics, Burgess Hill, UK) between 0 and $120 \mathrm{~min}$. Additional blood was collected during the IPGTT to estimate the insulin secretory response to glucose.

Model assessments of insulin resistance Whole-body insulin resistance and sensitivity were assessed using three equations: (1) insulin resistance $(\mathrm{IR})=$ fasting glucose $(\mathrm{mmol} / \mathrm{l}) \times$ fasting insulin (pmol/1); (2) HOMA-IR (log HOMA-IR $=\log _{10}$ [fasting insulin $(\mathrm{pmol} / \mathrm{l}) \times$ fasting glucose $(\mathrm{mmol} / \mathrm{l}) \div 22.5]$ ); and (3) QUICKI $=\left(1 / \log _{10}\right.$ [fasting insulin $\left.(\mathrm{pmol} / \mathrm{l})\right]+\log _{10}$ [fasting glucose $(\mathrm{mmol} / \mathrm{l})]$ ). 
Blood chemistry Blood was obtained by cardiac puncture. Serum and liver triacylglycerol levels were determined using a colourimetric assay kit (Cayman Chemical, Ann Arbor, MI, USA). Serum NEFA levels were measured using a fluorometric assay (Abcam, Cambridge, UK). ELISA kits were used to measure serum insulin (Mercodia, Uppsala, Sweden), eNAMPT (Caltag, Buckingham, UK), and TNF- $\alpha$, IL-1 $\beta$ and MCP1 (eBioscience, Hatfield, UK).

Serum NMN and tissue NAD levels Serum NMN was detected fluorometrically by HPLC, using a modified version of a previously described methodology (see electronic supplementary material [ESM] Methods) [16]. Tissue NAD levels were determined using a NAD/NADH quantification kit (Sigma).

Immunoblotting Immunoblotting was conducted as previously described [17] using primary antibodies against NAMPT (Sigma or Bethyl Laboratories, Montgomery, TX, USA), p-Akt $\left(\operatorname{Ser}^{473}\right.$ ) and total Akt (both Cell Signaling Technologies, Danvers, MA, USA). All primary antibodies were rabbit anti-mouse polyclonal antibodies and were used at a 1:1000 dilution. Validation was conducted via immunoblot. NAMPT immunoprecipitation was carried out using a Catch and Release Reversible Immunoprecipitation System (Millipore, Watford, UK).

Quantitative RT-PCR Gene expression was determined by TaqMan or SYBR green quantitative RT-PCR (qRT-PCR) [17], using $\Delta \Delta \mathrm{Ct}$ methodology, and normalised against $18 \mathrm{~S}$ ribosomal RNA levels (Applied Biosystems, Warrington, UK). Changes in gene expression are normalised to control. For details of primers (Eurogentec, Southampton, UK), see ESM Table 1

Mouse islet isolation and insulin secretion Mouse pancreases were digested in $2 \mathrm{ml}$ Hanks Buffered Salt Solution (HBSS) containing $1 \mathrm{mg} / \mathrm{ml}$ collagenase $\mathrm{P}$ and $0.15 \mathrm{mg} / \mathrm{ml}$ DNAse I (both Roche Diagnostics). Islets were hand-picked and transferred into RPMI 1640 medium for RNA extraction or insulin secretion assays. For islet insulin secretion assays, batches of eight size-matched islets were preincubated for $1 \mathrm{~h}$ at $37^{\circ} \mathrm{C}$ in $\mathrm{HBSS}$ containing $3 \mathrm{mmol} / 1$ glucose, $10 \mathrm{mmol} / 1$ HEPES (pH 7.4) and 0.2\% BSA (wt/ vol.). For glucose-stimulated insulin secretion (GSIS) analysis, islets were incubated for $1 \mathrm{~h}$ at $37^{\circ} \mathrm{C}$ in $\mathrm{HBSS}$ containing $10 \mathrm{mmol} / \mathrm{l} \mathrm{HEPES}(\mathrm{pH} 7.4$ ) and 0.2\% BSA, supplemented with $3 \mathrm{mmol} / 1$ or $17 \mathrm{mmol} / 1$ glucose. After $1 \mathrm{~h}$, the medium was collected for determination of insulin levels by ELISA (see ESM Methods 'islet isolation' and 'insulin secretion ex vivo' for further details).
Immunofluorescence analysis of mouse pancreatic sections Immunostaining was conducted as previously described [7]. Briefly, the whole pancreas was fixed in buffered formalin, embedded in paraffin, cut into sections and stained with guinea pig anti-insulin (1:100 dilution; Abcam) and/or rabbit antip-P38 (1:1600 dilution; Cell Signaling Technologies) antibodies. Sections were then mounted on glass cover slips and analysed using a Leica DM5000 epifluorescence microscope with Leica Application Suite software (Leica, Milton Keynes, UK) (see ESM Methods).

MIN6 cell culture and treatment MIN6 beta cells were incubated with 2-10 ng/ml eNAMPT (Adipogen) with or without anti-eNAMPT antibody $(2.5 \mu \mathrm{g} / \mathrm{ml}$; LS-C48964) and analysed for changes in insulin secretion or NAD levels (see ESM Methods). MIN6 cells were free from mycoplasma contamination

Isolation of white adipocytes and the stromal vascular fraction White adipocytes and the stromal vascular fraction (SVF) were isolated from epididymal white adipose tissue (AT) according to previously described methodology [18]. Isolated adipocyte and SVF preparations were incubated for $3.5 \mathrm{~h}$ at $37^{\circ} \mathrm{C}$ in DMEM containing $25 \mathrm{mmol} / 1$ glucose. The medium was then collected and analysed for eNAMPT content by ELISA (Caltag).

Statistical analysis Results are expressed as the mean \pm SEM. Statistical differences were calculated by one-way ANOVA and the Tukey's test where appropriate (GraphPad Software; la Jolla, CA, USA).

\section{Results}

High-fat feeding selectively induces the production and secretion of eNAMPT-monomer We first demonstrated that serum eNAMPT levels were elevated in diabetic HFD-fed mice (Fig. 1a).

Following this, we examined whether increased total eNAMPT was associated with specific changes in eNAMPTmonomer or eNAMPT-dimer levels using non-reducing SDS-PAGE and immunoblotting. Indicative of a selective diabetogenic function, eNAMPT-monomer protein levels were markedly elevated in serum $(82.0 \pm 1.9 \%$; Fig. $1 \mathrm{~b}, \mathrm{c})$ of HFD-fed mice compared with CON-fed mice. In contrast, serum levels of the eNAMPT-dimer and its reaction product, NMN, were non-significantly reduced or unchanged between HFD- vs CON-fed mice (Fig. 1b-d). Similar changes were observed in white AT, a major source of circulating eNAMPT. White AT from HFD-fed mice had increased Nampt mRNA levels (Fig. 1e), with parallel increases in the NAMPT-monomer $(64.0 \pm 5.9 \%)$, but no observed changes in 
Fig. 1 eNAMPT-monomer levels are selectively elevated in HFD-fed mice. (a) Total serum eNAMPT, $(\mathbf{b}, \mathbf{c})$ serum eNAMPT monomeric and dimeric protein and (d) serum NMN levels in CON- or HFD-fed mice (fed for 10 weeks); in (c) white bars, CON-fed; black bars, HFD-fed. (e) Nampt mRNA expression (normalised to control levels [levels in CON-fed mice]), (f, g) NAMPT-monomer and NAMPT-dimer protein levels (normalised to control [CONfed]) and (h) total NAD levels in white AT (in (c) and (g), white bars, CON-fed; black bars, HFDfed). eNAMPT secretion from (i) SVF and (j) white adipocytes. Western blots are representative of three separate blots. $* p<0.05$, $* * p<0.01$
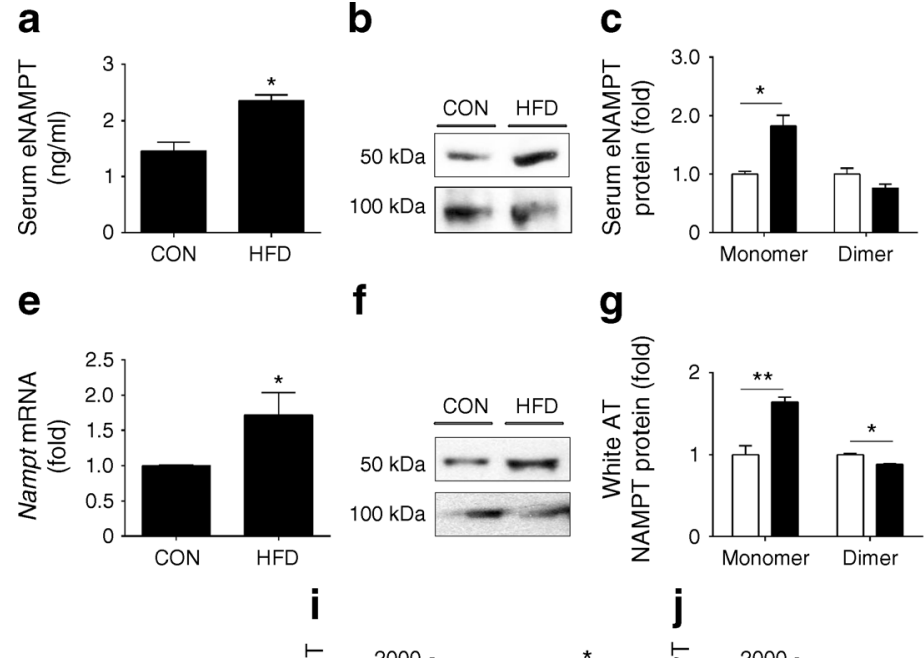

g
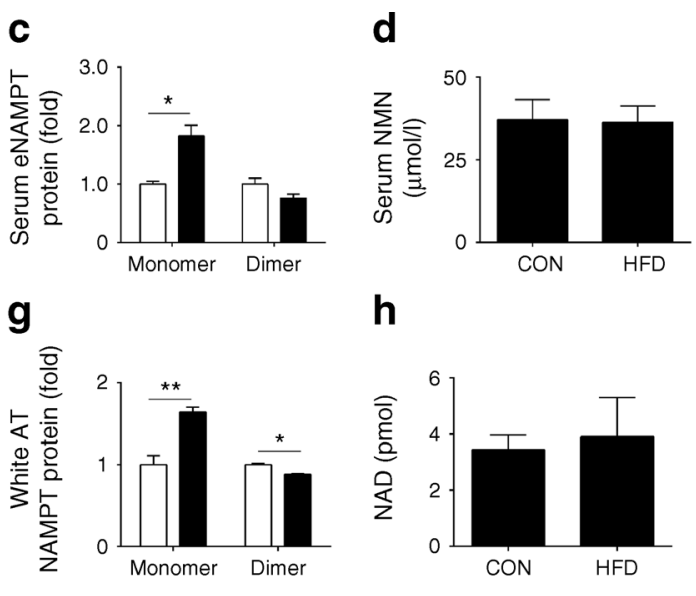

h

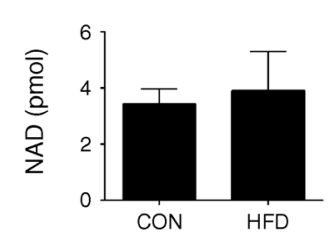

NAMPT-dimer or NAD (Fig. 1f-h). Therefore, alterations in the levels and structure of NAMPT in white AT provide a potential explanation for increased serum eNAMPTmonomer and eNAMPT-dimer levels in HFD-fed mice.

To further examine the source of eNAMPT, we isolated white adipocytes and SVF from epididymal white AT and measured eNAMPT secretion. In white adipocytes from HFD-fed mice eNAMPT secretion was markedly decreased, whilst eNAMPT secretion was increased in SVF isolated from HFDfed mice (Fig. 1i, j). Collectively, this suggests that white adipocytes and SVF are likely to be the primary source of eNAMPT-dimer and eNAMPT-monomer, respectively. The cellular source of eNAMPT-monomer within the SVF requires further study. However, previous studies have reported eNAMPT secretion from undifferentiated pre-adipocytes and immune cells, including macrophages, neutrophils and B cells.

Together these data demonstrate that serum levels of eNAMPT-monomer are selectively elevated in diabetic HFD-fed mice. This implies a specific pathophysiological role for eNAMPT-monomer in experimental diabetes.

Anti-Anti-eNAMPT antibody improves glycaemic control and insulin resistance in HFD-fed mice To determine the importance of raised serum eNAMPT-monomer levels in experimental diabetes, HFD- and CON-fed mice were injected i.p. with an anti-eNAMPT antibody (eNAMPT-Ab) or nonimmune IgG equivalent ( 2 doses/week in weeks 9-10). Immunoneutralisation enables the selective inhibition of circulating eNAMPT (without inhibiting iNAMPT). Immunoprecipitation of NAMPT with this antibody followed by immunoblotting led to detection of a $50 \mathrm{kDa}$ protein band but not a $100 \mathrm{kDa}$ protein band (ESM Fig. 1a, b), indicating antibody specificity for the eNAMPT-monomer. Moreover, eNAMPT-Ab blocked NAD-independent eNAMPT-mediated decreases in GSIS in MIN6 cells but did not have any effect on levels of NMN (the eNAMPT-dimer reaction product) in HFD- or CON-fed mice (ESM Fig. 1c, d). This result suggests that the eNAMPT-Ab selectively targets the eNAMPT-monomer. Serum levels of total eNAMPT were non-significantly reduced following eNAMPT-Ab treatment (ESM Fig. 1e).

In support of a diabetogenic role for the eNAMPT-monomer, eNAMPT-Ab administration reduced blood glucose and insulin levels (Fig. 2a-c) and corrected impaired glucose tolerance (IGT) in HFD-fed mice (Fig. 2d-f). As determined by assessment of the insulin $\times$ glucose product, HOMA-IR and QUICKI, eNAMPT-Ab also ameliorated whole-body insulin resistance (Fig. 2g-i). Improved glucose tolerance and insulin sensitivity were associated with reduced fasting serum triacylglycerol and fed serum NEFA levels, although the latter did not reach statistical significance. Fed serum triacylglycerol levels were unchanged across all groups (ESM Fig. 2a-c). Body weight and food intake were also unchanged (ESM Fig. 2d, e).

Together, these data demonstrate that neutralising the eNAMPT-monomer leads to a marked improvement in the diabetic phenotype of HFD-fed mice.

eNAMPT-Ab improves pancreatic islet function and increases islet size in HFD-fed mice Type 2 diabetes is characterised by progressive pancreatic beta cell failure [1] Therefore, we assessed whether eNAMPT-Ab mediated improvements in glycaemic control via improvements in beta cell/islet health. 
Fig. 2 eNAMPT-monomer immunoneutralisation reverses the diabetic phenotype in HFDfed mice. (a) Fasting serum glucose, (b) fed serum glucose, (c) fed serum insulin, (d-f) glucose response to IPGTT (black, $\mathrm{CON}^{\mathrm{IgG}}$; green, $\mathrm{CON}$-fed mice, administered eNAMPTantibody $\left[\mathrm{CON}^{\mathrm{Ab}}\right]$; red, $\mathrm{HFD}^{\mathrm{IgG}}$; blue, HFD ${ }^{\mathrm{Ab}}$ ) in CON- and HFDfed mice (fed for 10 weeks) treated with eNAMPT-Ab or nonimmune IgG. (g) Insulin $\times$ glucose product, (h) HOMA-IR and (i) QUICKI. * $p<0.05$, $* * p<0.01, * * * p<0.001$ for all subparts except (d) where ${ }^{\dagger} p<0.05,{ }^{\dagger} p<0.01,{ }^{\dagger \dagger} p<0.001$ for $\mathrm{CON}^{\mathrm{IgG}}$ vs HFD ${ }^{\mathrm{IgG}}$ and ${ }^{\star} p<0.05$ for $\mathrm{HFD}^{\mathrm{IgG}}$ vs HFD ${ }^{\mathrm{Ab}}$
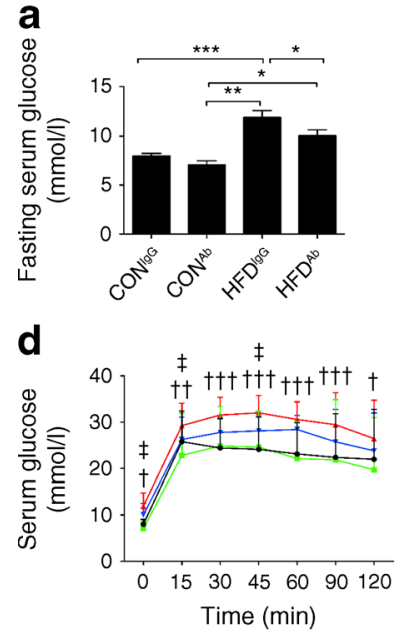

g

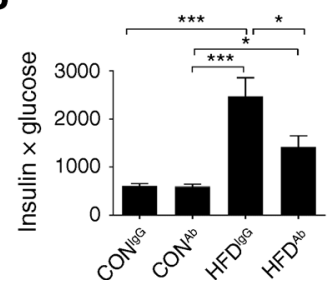

b

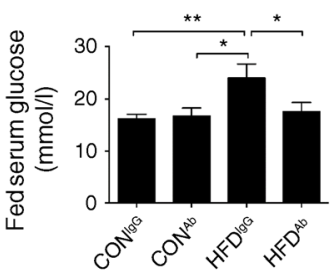

e

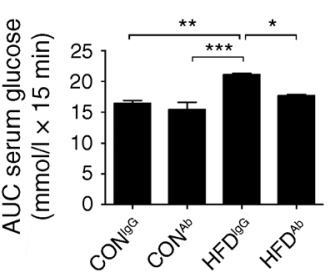

h

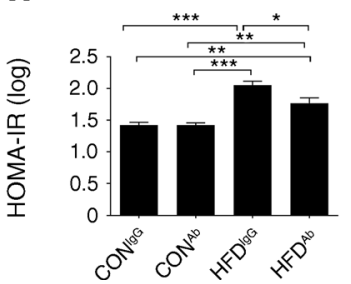

C

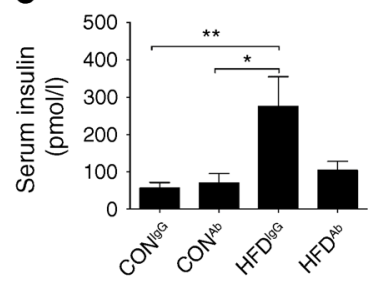

f

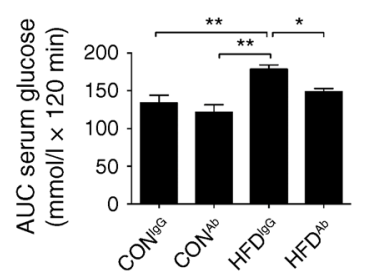

i

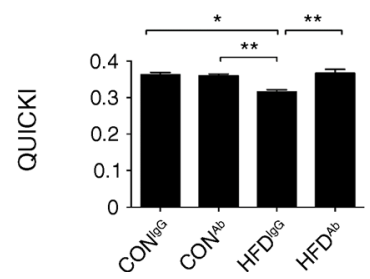

Beta cell/islet function was impaired in HFD-fed mice, as evidenced by the lack of an islet compensatory response to insulin resistance in static ex vivo GSIS studies (Fig. 3a), and in vivo measurements of the first (0-15 $\mathrm{min})$ and second phase (15-60 $\mathrm{min})$ insulin secretory responses to glucose (Fig. 3b, c). Crucially, eNAMPT immunoneutralisation restored islet compensation, as demonstrated by a marked increase in ex vivo GSIS and in vivo first phase insulin secretion in HFDfed mice, administered eNAMPT-antibody $\left(\mathrm{HFD}^{\mathrm{Ab}}\right)$.
Fig. 3 eNAMPT

immunoneutralisation reverses beta cell dysfunction in HFD-fed mice. (a) Ex vivo GSIS and (b, c) AUC insulin response to IPGTT in CON- and HFD-fed mice (fed for 10 weeks) i.p. injected with eNAMPT-Ab or non-immune IgG. (d) Insulin

immunofluorescence staining of whole pancreatic sections (magnification $\times 20)$, (e) relative islet size (AU, arbitrary unit) and (f) gene expression of proapoptotic genes in isolated islets (normalised to control $\left[\mathrm{CON}^{\mathrm{IgG}}\right]$ ). $(\mathbf{a}, \mathbf{f})$ white, $\mathrm{CON}^{\mathrm{IgG}}$; light grey, $\mathrm{CON}^{\mathrm{Ab}}$; black, $\mathrm{HFD}^{\mathrm{IgG}}$; dark grey, $\mathrm{HFD}^{\mathrm{Ab}} . * p<0.05$,

$* * p<0.01$ for all subparts except (f) where ${ }^{\dagger \dagger} p<0.001$ for $\mathrm{CON}^{\mathrm{IgG}}$ vs $\mathrm{HFD}^{\mathrm{IgG}},{ }^{\mathrm{H}} p<0.001$ for $\mathrm{HFD}^{\mathrm{IgG}}$ vs $\mathrm{HFD}^{\mathrm{Ab}}$ and ${ }^{\S \S} p<0.01$ for $\mathrm{CON}^{\mathrm{Ab}}$ vs HFD ${ }^{\mathrm{IgG}}$ a

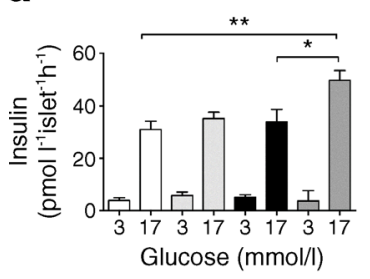

b
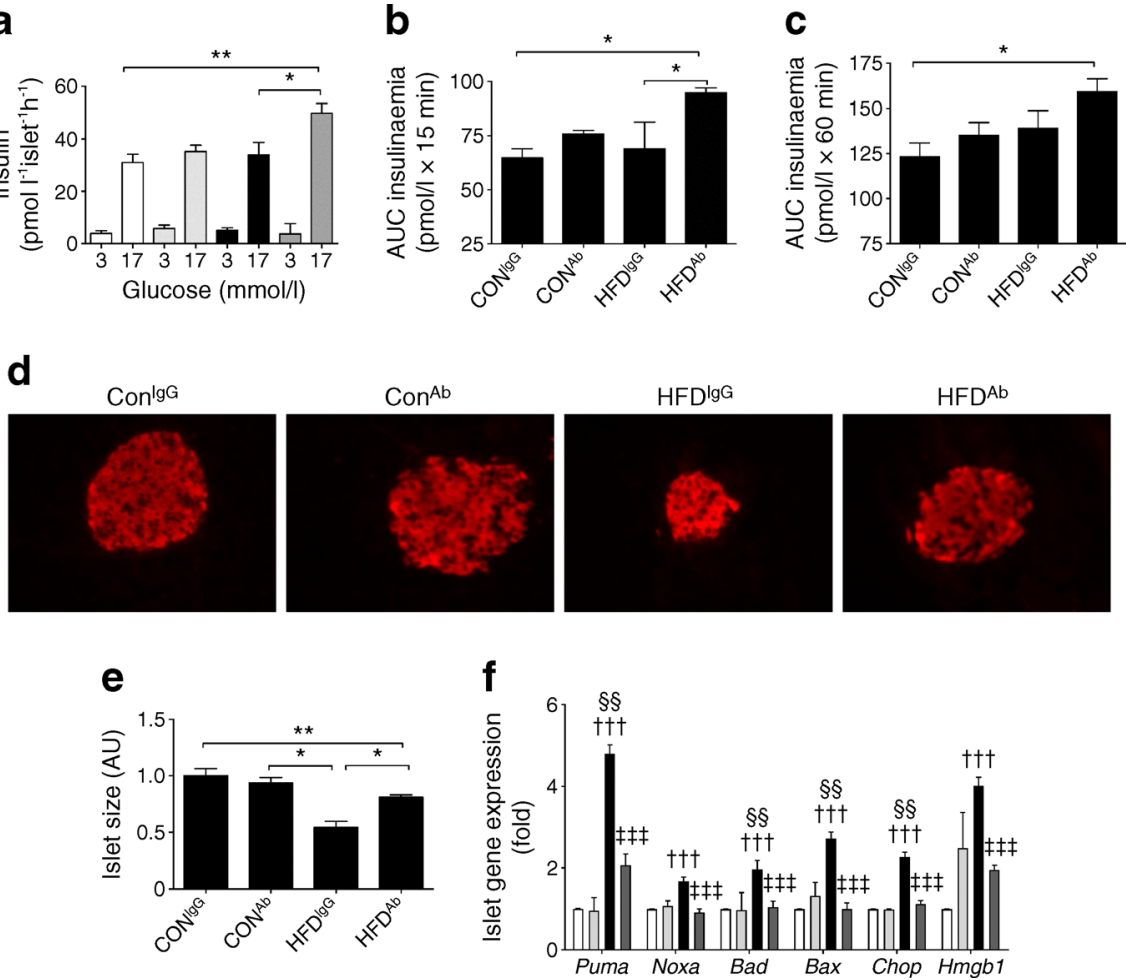
We next assessed the effects of the eNAMPT-Ab on islet size. Islet size was reduced by $46 \%(p<0.01)$ in HFD-fed mice, administered $\mathrm{IgG}\left(\mathrm{HFD}^{\mathrm{IgG}}\right)$ compared with $\mathrm{CON}$-fed mice, administered $\mathrm{IgG}\left(\mathrm{CON}^{\mathrm{IgG}}\right)$; this effect was completely reversed by eNAMPT-Ab treatment (Fig. 3d, e). Several studies have observed an increased islet size in HFD-fed mice. However, in this study the reduced islet size observed is likely to reflect progression from insulin resistance to an overt diabetic phenotype in our model. Beta cell apoptosis and necrosis are commonly considered to be mechanisms responsible for reduced beta cell/islet mass in type 2 diabetes. Consistent with this, islet mRNA levels of pro-apoptotic and necrotic markers were significantly elevated in HFD-fed mice (Fig. 3f). Strikingly, mRNA levels of these genes were lowered to basal levels following eNAMPT-Ab administration, demonstrating that eNAMPT immunoneutralisation improves beta cell function/ mass in part by protecting against beta cell apoptosis and necrosis. Together, these data demonstrate that eNAMPT immunoneutralisation improves glycaemic control in HFD-fed mice in part through reversing beta cell dysfunction and restoring islet compensation.

eNAMPT-Ab improves hepatic insulin sensitivity and reduces hepatic fat content Obesity-induced non-alcoholic fatty liver disease (NAFLD), characterised by excess hepatic lipid accumulation and inflammation, is an established risk factor for the development of hepatic insulin resistance and type 2 diabetes [19]. Elevated serum eNAMPT levels in NAFLD are associated with worsening disease severity [20]. Consistent with a potential role for eNAMPT-monomer in the development of fatty liver, the eNAMPT-Ab reversed HFD-mediated increases in hepatic triacylglycerol content (ESM Fig. 3a) and changes in Srebfl and Fasn lipogenic gene expression (ESM Fig. 3b). Hepatic insulin resistance is strongly associated with increased liver lipid content. Moreover, eNAMPT-Ab reversed HFD-mediated decreases in hepatic protein levels of -p-Akt $\left(\mathrm{Ser}^{473}\right)$, a marker of insulin signalling (ESM Fig. 3c), and corrected abnormally elevated mRNA levels of Pck1 (ESM Fig. 3b), a key gluconeogenic gene. Together, these data suggest that eNAMPT immunoneutralisation lowers hepatic lipid content and may improve hepatic insulin sensitivity in HFD-fed mice.

eNAMPT-Ab ameliorates tissue and systemic inflammation in HFD-fed mice Chronic inflammation causes beta cell failure in type 2 diabetes [21]. Proinflammatory functions of eNAMPT have been reported, although studies have not distinguished between monomeric and dimeric forms [10], nor have they examined a specific proinflammatory role for eNAMPT in type 2 diabetes. The eNAMPT-Ab lowered HFD-mediated increases in serum monocyte chemoattractant protein (MCP-1; also known as chemokine (C-C motif) ligand 2[CCL2]) levels (Fig. 4a), although serum TNF- $\alpha$ and IL-1 $\beta$ levels were similar in all experimental groups (Fig. 4b, c).
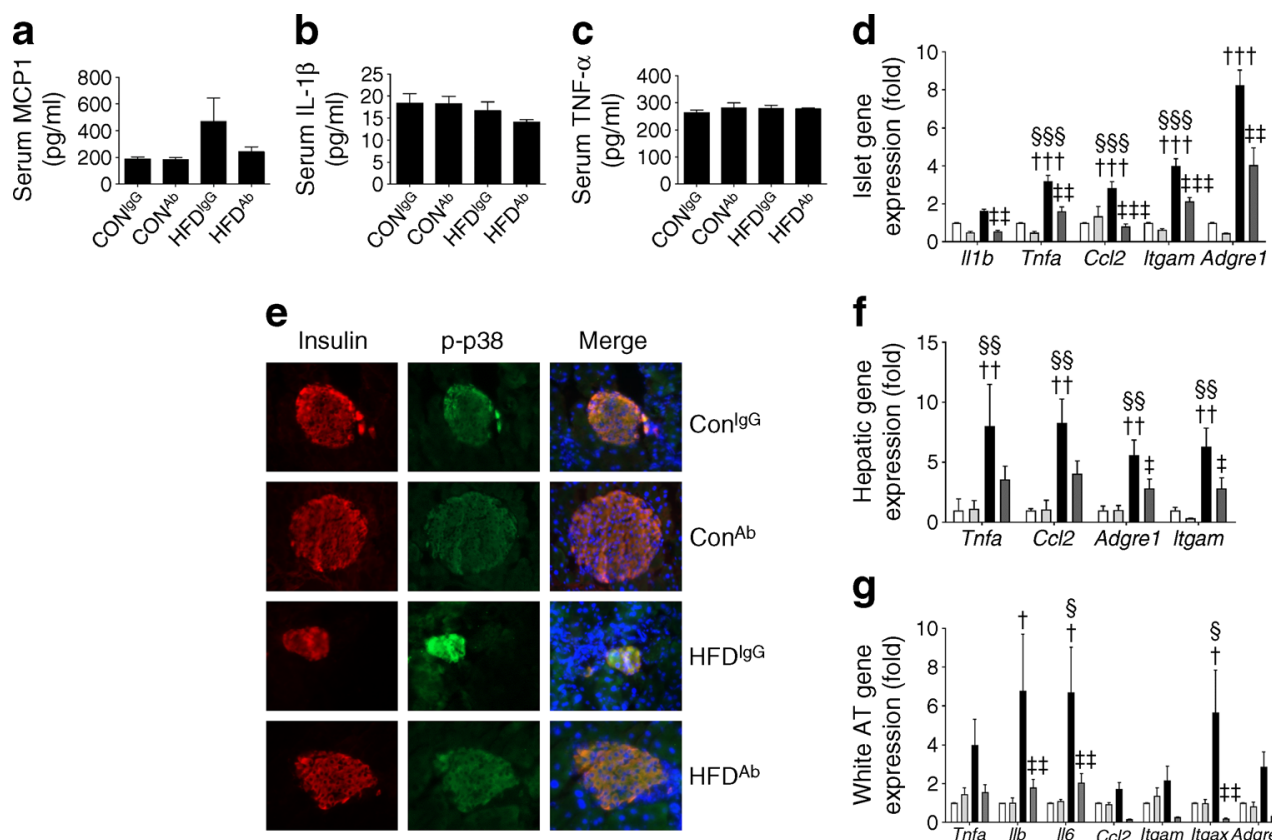

f
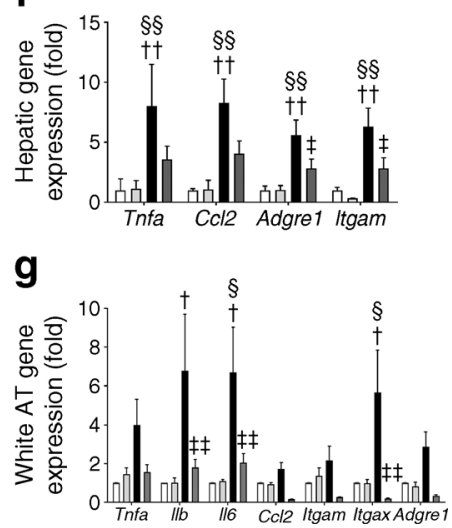

Fig. 4 eNAMPT-monomer immunoneutralisation improves tissue and systemic inflammation in HFD-fed mice. Serum concentrations of (a) MCP1, (b) IL-1 $\beta$ and (c) TNF- $\alpha$ in CON- and HFD-fed mice (fed for 10 weeks) injected i.p. with eNAMPT-Ab or non-immune IgG. (d) Gene expression of proinflammatory genes in isolated islets, (e) insulin and pp38 islet immunofluorescence staining of whole pancreatic sections

(magnification $\times 20$ ), $(\mathbf{f})$ hepatic expression of proinflammatory genes and (g) white AT proinflammatory gene expression (normalised to control $\left.\left[\mathrm{CON}^{\mathrm{IgG}}\right]\right)$. (e, f ) white, $\mathrm{CON}^{\mathrm{IgG}}$; light grey, $\mathrm{CON}^{\mathrm{Ab}}$; black, $\mathrm{HFD}^{\mathrm{IgG}}$; dark grey, $\mathrm{HFD}^{\mathrm{Ab}} .{ }^{\dagger} p<0.05,{ }^{\dagger \dagger} p<0.01$ and ${ }^{\dagger \dagger} p<0.001$ for $\mathrm{CON}^{\mathrm{IgG}}$ vs $\mathrm{HFD}^{\mathrm{IgG}} ;{ }^{\ddagger} p<0.05,{ }^{\star} p<0.01$ and ${ }^{\dagger+} p<0.001$ for HFD ${ }^{\mathrm{IgG}}$ vs HFD ${ }^{\mathrm{Ab}}$; and ${ }^{\S} p<0.05,{ }^{\S} p<0.01$ and ${ }^{\S \S} p<0.001$ for $\mathrm{CON}^{\mathrm{Ab}}$ vs $\mathrm{HFD}^{\mathrm{IgG}}$ 
Furthermore, eNAMPT-Ab reversed HFD-mediated increases in islet mRNA levels of proinflammatory cytokines ( Tnfa, $I l l b, I l O)$, chemokines (Ccl2, encoding MCP1) and immune cell markers (Itgam, encoding CD11b; Itgax, encoding CD11c; Adgre1, encoding F4/80; Fig. 4d). Studies in monocytes have reported that eNAMPT induces proinflammatory cytokine production in part through activation of p38 mitogen-activated protein kinase (MAPK) [10]. In agreement, we found that the islet p-p38 immunofluorescence signal (denoting activated p38) was enhanced in HFD-fed mice compared with $\mathrm{CON}$-fed mice, and that this effect was reversed by eNAMPT-Ab treatment (Fig. 4e). Chronic inflammation in liver and white AT is also common in obesity and type 2 diabetes, where it plays a crucial role in disease pathophysiology. Consistent with a proinflammatory effect for the eNAMPT-monomer in these tissues, eNAMPT-Ab lowered the expression of proinflammatory genes in the liver and AT of HFD-fed mice (Fig. 4f, g). These data provide strong support for the notion that eNAMPT immunoneutralisation improves pancreatic beta cell health and peripheral insulin resistance through resolution of inflammation in HFD-fed mice, and that the eNAMPT-monomer is likely to exert proinflammatory effects.

The beneficial effects of eNAMPT-Ab are maintained 3 weeks post-treatment To determine whether the effects of eNAMPT immunoneutralisation were maintained over time, eNAMPT-Ab was administered in weeks 9-10, as previously described. Mice then remained on CON or HFD diets until week 13 without further eNAMPT-Ab administration. At
3 weeks post-treatment, the eNAMPT-Ab-lowered HFDmediated increases in blood glucose enhanced islet insulin secretion, reduced islet inflammation, and reduced hepatic triacylglycerol content, hepatic lipogenic gene expression and hepatic inflammation (Fig. 5). Thus, the beneficial effects of a single dose regimen of eNAMPT-Ab were maintained for at least 3 weeks post treatment.

eNAMPT-monomer administration for 14 days induces a diabetic phenotype in mice Finally, we examined the impact of experimental elevation of serum eNAMPT-monomer levels on glycaemic control in mice. Lean, non-diabetic mice were injected i.p. with recombinant eNAMPT or the equivalent volume of $\mathrm{NaCl}$ (154 mmol/l). eNAMPT administration doubled serum eNAMPT levels to $3.05 \pm 0.37 \mathrm{ng} / \mathrm{ml}$ (Fig. 6a), similar to levels in HFD-fed mice. When recombinant eNAMPT protein was analysed by non-reducing SDS-PAGE and immunoblotting, a $50 \mathrm{kDa}$ protein band was detected but a $100 \mathrm{kDa}$ protein band was not (ESM Fig. 1f). Moreover, recombinant eNAMPT treatment did not increase NAD levels in vitro in MIN6 cells, nor in vivo in mouse white AT (ESM Fig. 1g, h). These findings suggest that the recombinant eNAMPT used in this study does not dimerise to promote NAD biosynthesis, and thus represents monomeric eNAMPT. Therefore, to mimic elevated dimeric eNAMPT, a separate group of mice were injected i.p. with NMN, the reaction product of the eNAMPT-dimer's NAD biosynthetic reaction.

The 14-day eNAMPT-monomer administration resulted in elevated fasting blood glucose levels (Fig. 6b), development of IGT (0-30 min post-glucose injection; Fig 6c) and whole- a

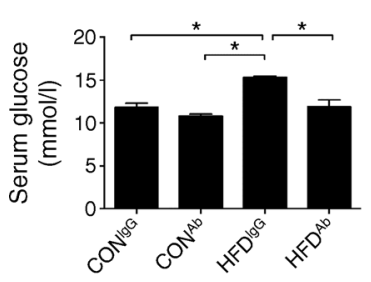

d

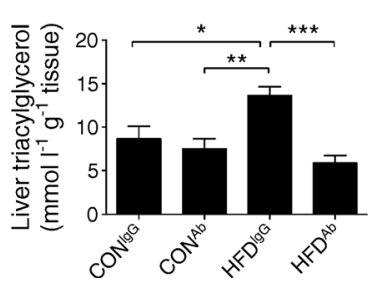

Fig. 5 The beneficial effects of eNAMPT-monomer immunoneutralisation in HFD-fed mice are maintained for 3 weeks post-treatment. (a) Fed serum glucose levels, (b) ex vivo GSIS, (c) islet expression of proinflammatory genes, (d) hepatic triacylglycerol levels, (e) hepatic expression of lipogenic and gluconeogenic genes and (f) hepatic expression of proinflammatory genes in CON- and HFD-fed mice (fed for 13 weeks), injected i.p. with b

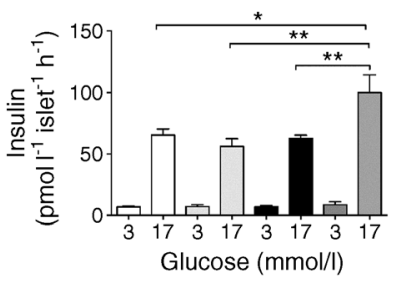

e

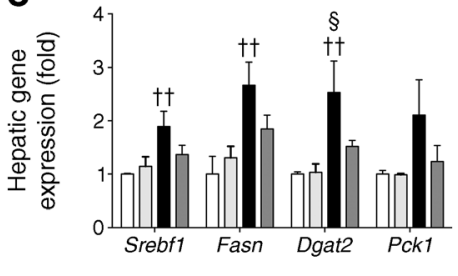

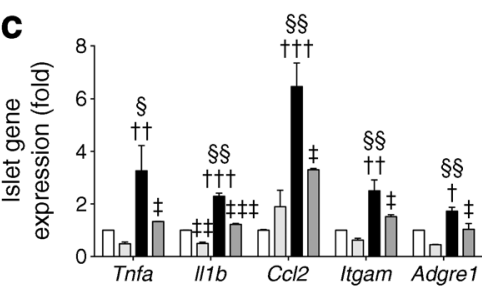

f

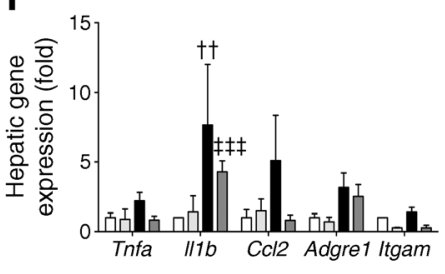

eNAMPT-Ab or non-immune IgG (at weeks 9-10; c, e, f: normalised to control $\left.\left[\mathrm{CON}^{\mathrm{IgG}}\right]\right)$. In (b, c, e, f), white, $\mathrm{CON}^{\mathrm{IgG}}$; light grey, $\mathrm{CON}^{\mathrm{Ab}}$; black, $\mathrm{HFD}^{\mathrm{IgG}}$; dark grey, HFD ${ }^{\mathrm{Ab}} . * p<0.05$; ** $p<0.01, * * * p<0.001$ for all subparts except (c, e, f) where ${ }^{\dagger} p<0.05,{ }^{\dagger} p<0.01$ and ${ }^{\dagger \dagger} p<0.001$ for $\mathrm{CON}^{\mathrm{IgG}}$ vs HFD ${ }^{\mathrm{IgG}},{ }^{\star} p<0.05,{ }^{\star} p<0.01,{ }^{\mathrm{*}} p<0.001$ for $\mathrm{HFD}^{\mathrm{IgG}}$ vs $\mathrm{HFD}^{\mathrm{Ab}}$ and ${ }^{\S} p<0.05$ and ${ }^{\S} p<0.01$ for $\mathrm{CON}^{\mathrm{Ab}}$ vs $\mathrm{HFD}^{\mathrm{IgG}}$ 
Fig. 6 eNAMPT-monomer administration for 14 days induces a diabetic phenotype in mice. (a) Serum eNAMPT levels, (b) blood glucose levels, (c) IPGTT (squares, saline; triangles, eNAMPT),

(d) QUICKI and (e) HOMA-IR in mice injected i.p. with recombinant eNAMPT-monomer or the equivalent volume of $154 \mathrm{mmol} / \mathrm{l} \mathrm{NaCl}$. AUC insulin response to IPGTT at (f) $0-15 \mathrm{~min}$ and (g) 15-60 $\mathrm{min}$, (h) serum MCP1 levels and (i) $C c l 2$ gene expression (white bars, saline; black bars, eNAMPT). ${ }^{*} p<0.05$, $* * p<0.01$
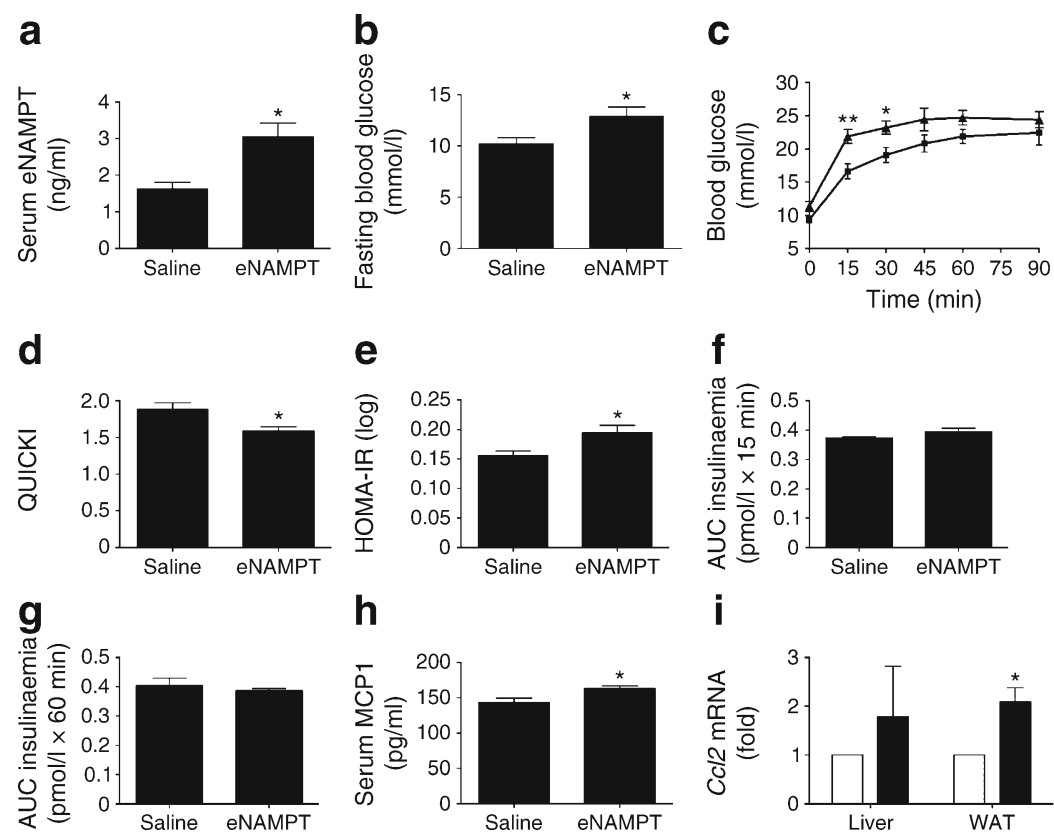

i

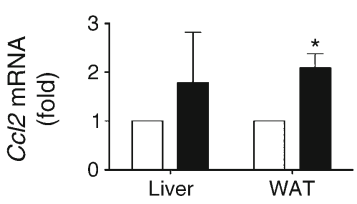

body insulin resistance, as determined by QUICKI and HOMA-IR (Fig. 6d, e). These changes were not related to changes in body weight, serum triacylglycerol or insulin levels (ESM Fig. 4a-d). Moreover, eNAMPT-monomer administration led to impaired insulin secretion, as demonstrated by a lack of islet compensatory response to insulin resistance (Fig. 6f, g). Consistent with its hypothesised proinflammatory actions, eNAMPT-monomer administration led to elevated serum of MCP1 levels (Fig. 6h) and increased liver and white AT $C c l 2$ mRNA levels (Fig. 6i), although serum IL- $1 \beta$ and TNF- $\alpha$ levels and liver and white AT mRNA levels of a number of other proinflammatory markers remained unchanged (ESM Fig. 4e-h). eNAMPT-monomer administration induced a nonsignificant trend towards increased proinflammatory gene expression in islets (ESM Fig. 4i).

In contrast to the diabetogenic effects of the eNAMPTmonomer, NMN administration resulted in mild reductions in blood glucose and serum IL-1 $\beta$ levels, without changes in body weight, glucose tolerance or insulin sensitivity (ESM Fig. 5).

Together, these data suggest distinct structure-function characteristics of eNAMPT monomers and dimers; the eNAMPT-monomer induced a diabetic phenotype in mice via NAD-independent proinflammatory effects, whilst eNAMPT-dimer/NMN elevation led to mild improvements in glycaemic control.

\section{Discussion}

We provide multiple lines of evidence suggesting an important NAD-independent role for the eNAMPT-monomer in type 2 diabetes: (1) diabetic HFD-fed mice displayed a selective increase in serum eNAMPT-monomer levels; (2) blocking eNAMPT action reversed the diabetogenic effects of HFD; and (3) 14-day administration of the eNAMPT-monomer induced a diabetic phenotype in non-diabetic mice.

Elevated serum eNAMPT levels are reported in type 2 diabetes [2, 3, 20, 22-24], although other studies have observed decreased or unchanged serum eNAMPT levels in metabolic diseases $[25,26]$.

Our findings go some way in clarifying the contradictory findings regarding eNAMPT and type 2 diabetes by demonstrating differences in the levels of eNAMPT-monomer and eNAMPT-dimer in experimental diabetes and highlighting the relevance of structure-function differences between monomer and dimer.

The precise function of eNAMPT and the relationship between raised eNAMPT levels and type 2 diabetes pathophysiology remain unresolved. Previous experimental studies have demonstrated both beneficial and deleterious effects of recombinant eNAMPT on insulin secretion and sensitivity in vitro [27-30]. However, these studies have often examined the acute effects of eNAMPT, which are unlikely to accurately represent a diabetic phenotype, or have used supraphysiological concentrations of eNAMPT. Crucially, these studies also did not distinguish between eNAMPT-monomers and eNAMPT-dimers.

Our studies, and those of other groups, have described beneficial acute and chronic effects of NMN, the reaction product of the eNAMPT-dimer biosynthetic reaction $[4,6,7,31,32]$. Consistent with a beneficial effect of the eNAMPT-dimer, serum and white AT eNAMPT-dimer levels were unchanged or decreased in HFD-fed mice, whilst 14-day NMN administration lowered blood glucose and inflammation in non- 
diabetic mice. In contrast, we describe a specific role for the eNAMPT-monomer in experimental diabetes via functioning partly through NAD-independent proinflammatory effects. Proinflammatory effects of eNAMPT in type 2 diabetes have not previously been reported. However, our findings are broadly in agreement with studies of eNAMPT in other diseases, although such studies did not distinguish between monomers and dimers. For example, eNAMPT is reported to induce monocyte expression and secretion of IL- 6 , IL- $1 \beta$ and TNF- $\alpha[10]$, potentially via receptor-mediated mechanisms [33]. Thus, normalisation of eNAMPT-monomer signalling in type 2 diabetes would be predicted to resolve inflammationmediated beta cell dysfunction and insulin resistance and improve glycaemic control.

We hypothesise that increased monomer levels are partly related to AT dysfunction. AT-derived eNAMPT-dimer is secreted from fully differentiated adipocytes, whilst the monomer may be secreted from poorly-differentiated adipocytes and immune cells $[5,9,10,34-36]$. Since poor adipocyte differentiation and immune cell infiltration are characteristics of obese AT [37], we hypothesise that such pathophysiological changes could explain the increased monomer levels in HFD-fed mice. In agreement, eNAMPT secretion from the AT SVF (which contains immune cells and undifferentiated pre-adipocytes) was markedly increased in HFD-fed mice. Thus, we hypothesise that obesity-mediated AT dysfunction results in a phenotypic switch, characterised by elevated eNAMPT-monomer production from SVF and reduced dimer secretion from adipocytes. Future studies will elucidate the precise mechanisms involved in dimer formation and identify the main cellular source of eNAMPT within the SVF.

Together, these studies suggest that the eNAMPTmonomer is an attractive therapeutic target for type 2 diabetes. Future strategies to develop this therapeutic approach will include development of eNAMPT-monomer receptor antagonists, specific eNAMPT-monomer inhibitors and humanised eNAMPT monoclonal antibodies. Humanised monoclonal antibodies can be engineered with an extended $t^{1} / 2$, potentially allowing clinical benefits to be achieved with only one dose every 1-3 months [38], thus providing benefits in terms of cost, convenience and compliance.

In summary, we have demonstrated that elevated eNAMPTmonomer levels contribute to the development of type 2 diabetes. In addition, we have provided proof-of-concept evidence that selectively blocking the action of eNAMPT-monomer is a promising therapeutic strategy for the treatment of type 2 diabetes.

Acknowledgements We are grateful to J. I. Miyazaki (University of Osaka, Osaka, Japan) for the provision of MIN6 beta cells. Some of the data in this manuscript were presented in abstract form at the ADA Scientific Sessions meeting in 2015 and at the Diabetes UK professional conference meeting in 2016.
Funding This work was supported by the following funding from the European Foundation for the Study of Diabetes/Lilly fellowship, a Society for Endocrinology Early Career Grant and a Diabetes UK project grant (15/0005154) to PWC. MFS was funded by FUNDACAO PARA A CIENCIA E TECNOLOGIA, Ministerio da Educacao e Ciencia, Portugal. JK, SMH and MMY are supported by the Bart's and the London National Institute of Health Research Cardiovascular Biomedical Research Unit.

Duality of interest statement The authors declare that there is no duality interest associated with this manuscript.

Contribution statement $\mathrm{PWC}$ is responsible for the integrity of the work as a whole. JK, SRS, MFS, SMH, MMY and PWC designed the research, analysed data and reviewed and approved the final manuscript; JK, SRS, MFS, SMH and PWC performed research; and PWC wrote the paper.

Open Access This article is distributed under the terms of the Creative Commons Attribution 4.0 International License (http:// creativecommons.org/licenses/by/4.0/), which permits unrestricted use, distribution, and reproduction in any medium, provided you give appropriate credit to the original author(s) and the source, provide a link to the Creative Commons license, and indicate if changes were made.

\section{References}

1. DeFronzo RA, Abdul-Ghani MA (2011) Preservation of beta-cell function: the key to diabetes prevention. J Clin Endocrinol Metab 96:2354-2366

2. Chang YH, Chang DM, Lin KC, Shin SJ, Lee YJ (2011) Visfatin in overweight/obesity, type 2 diabetes mellitus, insulin resistance, metabolic syndrome and cardiovascular diseases: a meta-analysis and systemic review. Diabetes Metab Res Rev 27:515-527

3. Lopez-Bermejo A, Chico-Julia B, Fernandez-Balsells M et al (2006) Serum visfatin increases with progressive beta-cell deterioration. Diabetes 55:2871-2875

4. Yoshino J, Mills KF, Yoon MJ, Imai S (2011) Nicotinamide mononucleotide, a key $\mathrm{NAD}(+)$ intermediate, treats the pathophysiology of diet- and age-induced diabetes in mice. Cell Metab 14:528-536

5. Revollo JR, Korner A, Mills KF et al (2007) Nampt/PBEF/Visfatin regulates insulin secretion in beta cells as a systemic NAD biosynthetic enzyme. Cell Metab 6:363-375

6. Caton PW, Kieswich J, Yaqoob MM, Holness MJ, Sugden MC (2011) Nicotinamide mononucleotide protects against proinflammatory cytokine-mediated impairment of mouse islet function. Diabetologia 54:3083-3092

7. Caton PW, Richardson SJ, Kieswich J et al (2013) Sirtuin 3 regulates mouse pancreatic beta cell function and is suppressed in pancreatic islets isolated from human type 2 diabetic patients. Diabetologia 56:1068-1077

8. Imai S (2009) Nicotinamide phosphoribosyltransferase (Nampt): a link between NAD biology, metabolism, and diseases. Curr Pharm Des 15:20-28

9. Samal B, Sun Y, Stearns G, Xie C, Suggs S, McNiece I (1994) Cloning and characterization of the cDNA encoding a novel human pre-B cell colony-enhancing factor. Mol Cell Biol 14:1431-1437

10. Moschen AR, Kaser A, Enrich B et al (2007) Visfatin, an adipocytokine with proinflammatory and immunomodulating properties. J Immunol 178:1748-1758

11. Fukuhara A, Matsuda M, Nishizawa M et al (2005) Visfatin: a protein secreted by visceral fat that mimics the effects of insulin. Science 307:426-430 
12. Rongvaux A, Shea RJ, Mulks MH et al (2002) Pre-B cell colonyenhancing factor, whose expression is up-regulated in activated lymphocytes, is a nicotinamide phosphoribosyltransferase, a cytosolic enzyme involved in NAD biosynthesis. Eur J Immunol 32:3225-3234

13. Yoon MJ, Yoshida M, Johnson S et al (2015) SIRT1-mediated eNAMPT secretion from adipose tissue regulates hypothalamic NAD and function in mice. Cell Metab 21:705-717

14. Hara N, Yamada K, Shibata T, Osago H, Tsuchiya M (2011) Nicotinamide phosphoribosyltransferase/visfatin does not catalyze nicotinamide mononucleotide formation in blood plasma. PLoS One 6:e22781

15. Wang $\mathrm{T}$, Zhang $\mathrm{X}$, Bheda $\mathrm{P}$, Revollo JR, Imai $\mathrm{S}$, Wolberger $\mathrm{C}$ (2006) Structure of Nampt/PBEF/visfatin, a mammalian NAD+ biosynthetic enzyme. Nat Struct Mol Biol 13:661-662

16. Formentini L, Moroni F, Chiarugi A (2009) Detection and pharmacological modulation of nicotinamide mononucleotide (NMN) in vitro and in vivo. Biochem Pharmacol 77:1612-1620

17. Caton PW, Nayuni NK, Kieswich J, Khan NQ, Yaqoob MM, Corder R (2010) Metformin suppresses hepatic gluconeogenesis through induction of SIRT1 and GCN5. J Endocrinol 205:97-106

18. Weisberg SP, McCann D, Desai M, Rosenbaum M, Leibel RL, Ferrante AW Jr (2003) Obesity is associated with macrophage accumulation in adipose tissue. J Clin Invest 112:1796-1808

19. Birkenfeld AL, Shulman GI (2014) Nonalcoholic fatty liver disease, hepatic insulin resistance, and type 2 diabetes. Hepatology 59:713-723

20. Moschen AR, Gerner RR, Tilg H (2010) Pre-B cell colony enhancing factor/NAMPT/visfatin in inflammation and obesity-related disorders. Curr Pharm Des 16:1913-1920

21. Donath MY, Shoelson SE (2011) Type 2 diabetes as an inflammatory disease. Nat Rev Immunol 11:98-107

22. Chen MP, Chung FM, Chang DM et al (2006) Elevated plasma level of visfatin/pre-B cell colony-enhancing factor in patients with type 2 diabetes mellitus. J Clin Endocrinol Metab 91:295-299

23. Haider DG, Holzer G, Schaller G et al (2006) The adipokine visfatin is markedly elevated in obese children. J Pediatr Gastroenterol Nutr 43:548-549

24. Krzyzanowska K, Krugluger W, Mittermayer F et al (2006) Increased visfatin concentrations in women with gestational diabetes mellitus. Clin Sci 110:605-609

25. Pagano C, Pilon C, Olivieri M et al (2006) Reduced plasma visfatin/ pre-B cell colony-enhancing factor in obesity is not related to insulin resistance in humans. J Clin Endocrinol Metab 91:3165-3170

26. Berndt J, Kloting N, Kralisch S et al (2005) Plasma visfatin concentrations and fat depot-specific mRNA expression in humans. Diabetes 54:2911-2916
27. da Kim S, Kang S, Moon NR, Park S (2014) Central visfatin potentiates glucose-stimulated insulin secretion and beta-cell mass without increasing serum visfatin levels in diabetic rats. Cytokine 65:159-166

28. Cheng Q, Dong W, Qian L, Wu J, Peng Y (2011) Visfatin inhibits apoptosis of pancreatic beta-cell line, MIN6, via the mitogenactivated protein kinase/phosphoinositide 3-kinase pathway. J Mol Endocrinol 47:13-21

29. Brown JE, Onyango DJ, Ramanjaneya M et al (2010) Visfatin regulates insulin secretion, insulin receptor signalling and mRNA expression of diabetes-related genes in mouse pancreatic beta-cells. J Mol Endocrinol 44:171-178

30. Oita RC, Ferdinando D, Wilson S, Bunce C, Mazzatti DJ (2010) Visfatin induces oxidative stress in differentiated $\mathrm{C} 2 \mathrm{C} 12$ myotubes in an Akt-and MAPK-independent, NFkB-dependent manner. Pflugers Arch 459:619-630

31. Ramsey KM, Mills KF, Satoh A, Imai S (2008) Age-associated loss of Sirt1-mediated enhancement of glucose-stimulated insulin secretion in beta cell-specific Sirt1-overexpressing (BESTO) mice. Aging Cell 7:78-88

32. Spinnler R, Gorski T, Stolz K et al (2013) The adipocytokine Nampt and its product NMN have no effect on beta-cell survival but potentiate glucose stimulated insulin secretion. PLoS One 8: e54106

33. Camp SM, Ceco E, Evenoski CL et al (2015) Unique Toll-like receptor 4 activation by NAMPT/PBEF induces NFkB signaling and inflammatory lung injury. Sci Rep 5:13135

34. Friebe D, Neef M, Kratzsch J et al (2011) Leucocytes are a major source of circulating nicotinamide phosphoribosyltransferase (NAMPT)/pre-B cell colony (PBEF)/visfatin linking obesity and inflammation in humans. Diabetologia 54:1200-1211

35. Dahl TB, Yndestad A, Skjelland M et al (2007) Increased expression of visfatin in macrophages of human unstable carotid and coronary atherosclerosis: possible role in inflammation and plaque destabilization. Circulation 115:972-980

36. Curat CA, Wegner V, Sengenes C et al (2006) Macrophages in human visceral adipose tissue: increased accumulation in obesity and a source of resistin and visfatin. Diabetologia 49:744747

37. Sun K, Kusminski CM, Scherer PE (2011) Adipose tissue remodeling and obesity. J Clin Invest 121:2094-2101

38. Boni-Schnetzler M, Donath MY (2013) How biologics targeting the IL-1 system are being considered for the treatment of type 2 diabetes. Br J Clin Pharmacol 76:263-268 